\title{
Vascular anatomical features of the medial thigh flap in human cadavers of Caucasian origin
}

\author{
M. Edızer ${ }^{1}$, A.O. Magden'1, V. Tayfur ${ }^{2}$, G.N. Yonguc ${ }^{1}$, N. Gocmen-Mas \\ ${ }^{1}$ Department of Anatomy, Faculty of Medicine, Dokuz Eylül University, Izmir, Turkey \\ ${ }^{2}$ Dr. Volkan Tayfur Estetik Klinik, Ufuk Üniversitesi Caddesi, Çankaya, Ankara, Turkey
}

[Received: 13 May 2015; Accepted: 10 June 2015]

Background: Medial fasciocutaneous flaps, which are based on the femoral artery from the thigh region, are used for wide inguinal, scrotal, vaginal, perineal, leg, head and neck defect reconstructions in injured human patients. Within this regard, anatomical knowledge about perforating and cutaneous branches of the femoral artery is important for the surgeons.

Materials and methods: In the present study, vascular pedicles of the medial thigh perforator flap based on the femoral artery were investigated according to anatomical and surgical landmarks. Human Caucasian preserved cadavers of 15 adults (13 males, 2 females; age range 55-82 years: 30 sides, bilaterally) that were previously formalin fixed were subjected to our analytical examinations. Micro dissections were performed under $4 \times$ loop magnification while representing the perforating branches of the femoral artery after filling by coloured latex injection via the external iliac artery.

Results: The size and length parameters of these branches which appeared around the apex of the femoral triangle were evaluated. The mean size of the perforating branch at the point of origin was $0.14 \mathrm{~cm}$ and the mean size of the cutaneous branch at the point of origin was $0.09 \mathrm{~cm}$, the mean length of the pedicle was $4.74 \mathrm{~cm}$ and the mean length of the cutaneous branch was $3.30 \mathrm{~cm}$, respectively. Location of the perforating and the cutaneous branches were also determined according to the surgical landmarks such as the anterior superior iliac spine, inguinal ligament, pubic tubercle and interepicondylar line.

Conclusions: The pedicle of the medial flap should locate up to $25 \mathrm{~cm}$ from the anterior superior iliac spine so as to preserve the vascular structures. Exact location of this artery helps the surgeons to perform anastomosis in an easier and safer manner during surgical operations. (Folia Morphol 2016; 75, 1: 27-32)

Key words: medial thigh flap, vascular pedicle, femoral artery, reconstruction

\section{INTRODUCTION}

The medial thigh flaps based on the femoral artery have favourable characteristics for use in extensive head, neck, thigh, leg and perinea defect surgical operations. The fasciocutaneous flaps from the medial thigh are proposed for vagina and perinea reconstru- ction in addition to the correction of the major scrotal defects including Fornier's gangrene in exposed testes and/or spermatic cords as well as post-burn groin contractures and malign tumour excision. Flaps are also used in defects after removal of skin cancer in the lower extremity and wide defects after resection

Address for correspondence: Ass. Prof. N. Gocmen-Mas, MD, PhD, Dokuz Eylül University, Faculty of Medicine, Department of Anatomy, Inciraltı Yerleşkesi, Izmir, Turkey, tel: + 90-(0)232-4124372, e-mail: nuketmas@gmail.com 
of the head and neck cancers, in addition to the scar repairing, labial separation, and flap transfer combined with liposuction $[5,8,10-12,16,19-21]$. The possibility of elevating and moving fasciocutaneous flaps from the medial thigh based on perforators from the femoral artery were firstly described by Beak [1] who defined the anatomy of the vasculature of the medial thigh flap [1, 14].

As recently described by Benninger [2] there is some terminological inconsistency or disagreement about the branching pattern of the femoral artery [15]. In order to overcome this issue we used the classical anatomical terminology in order to describe the femoral artery and its branches to medial thigh flap pedicle.

Prevention of the femoral artery and its perforating branch (PB) and cutaneous branch (CB) with further finding of the variations of the artery or its branches including the understanding of the anatomical characteristics of the adjacent to thigh region is important for the surgeons. Because of that we investigated the anatomical features of the femoral artery and its $\mathrm{PB}$ and $\mathrm{CB}$ in addition to their possible variations in details. We believe that all the findings of our study provide necessary information to the surgeons for better performance during corrective surgical operations.

\section{MATERIALS AND METHODS}

Dissections of a group of Caucasian cadavers previously fixed and preserved in formalin, including 13 males and 2 females with an age range from 55 to 82 years, were examined. Body heights were measured in centimetres using a steel measuring tape. The cadavers were placed on the dissection table in supine position. Then, the body heights of each cadaver were measured from the cranial vertex to the base of the heel.

After measuring body heights of each cadaver, the 2 extremities were examined, making a total number of 30 extremities subjected to analysis, which was performed under 4 times magnification via a loop. The measurements were taken by using a fine micro calliper and expressed in centimetres. Meticulous tangential micro-dissections of the thigh region of each cadaver were performed with following fasciocutaneous flaps elevation. The external iliac and femoral arteries were identified via dissecting and coloured latex that was injected to each external iliac artery. The vascular pedicle of medial thigh flap (VPMTF) and its muscular branch and CB were presented by dissecting the skin, the subcutaneous fat tissue, the deep fascia and the muscular layers, respectively.

The fallowing measurements regarding the VPMTF or in other words the $\mathrm{PB}$ and its $\mathrm{CB}$ were taken:

- The diameter of the PB at the origin point.

- The length of the pedicle.

- The diameter of the CB which is a fasciacutaneous perforator that pierces the fascia lata in order to supply the skin at its origin.

The origin and the course of the PB were examined as:

- The distance between the origin point of the PB and the anterior superior iliac spine.

- The distance between the origin point of the PB and the inguinal ligament.

- The distance between the origin point of the PB and the pubic tubercle.

The location of the origin of the perforator $C B$, which is originated from the VPMTF, at the piercing point of fascia lata was examined:

- The distance between the piercing point of $C B$ and the anterior superior iliac spine.

- The distance between the emerging point of the $\mathrm{CB}$ and the interepicondylar line.

- The distance between the emerging point of the $\mathrm{CB}$ and the public tubercle.

Further, accompanying veins and nerves were also noted.

\section{RESULTS}

\section{Observation of the medial thigh flap}

The VPMTF was observed (Figs. 1, 2). We found that the pedicle was appearing approximately in the apex of the femoral triangle which was shaped by the inguinal ligament, adductor longus and sartorius muscles. The unnamed vascular pedicle was originating from the femoral artery in all of the cases. The apex of femoral triangle may be noted as the anatomical landmark indicating the location of the flap perforators proximal to the adductor canal from the medial side of the femoral artery on anterior thigh region (Fig. 1). The VPMTF was giving muscular branches to sartorius muscle, adductor longus muscle and gracilis muscle and was giving fasciacutaneous PB which was piercing the fascia lata to supply the covering skin (Figs. 1, 2). Great saphenous vein was also noted to be located in the anterior to the vascular pedicle each of all cases. An accompanying 1 or 2 in nominate 


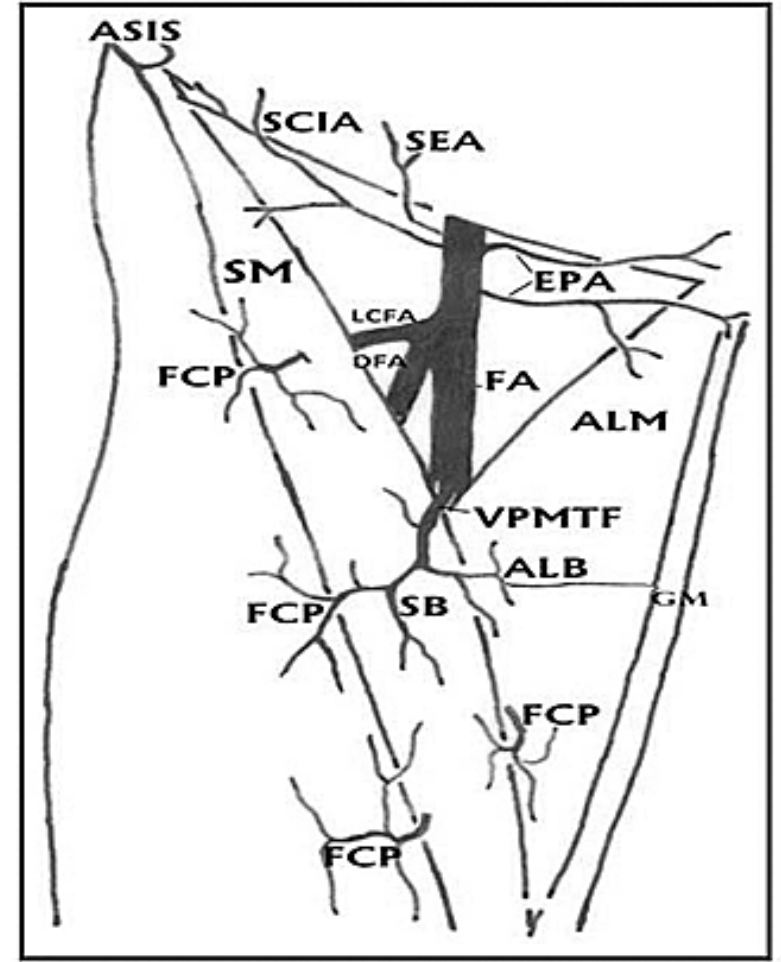

Figure 1. Anterior aspect of the thigh representing the vascular pedicle of medial thigh flap; FA — femoral artery; ALM — adductor longus muscle; GM — gracilis muscle; FCP — fasciocutaneous perforators around the sartorius muscle; DFA - deep femoral artery; LCFA — lateral circumflex femoral artery; VPMTF — vascular pedicle of the medial thigh fasciocutaneous flap; and its muscular and cutaneous branches; ALB - adductor longus branch to adductor longus muscle; $\mathrm{SB}$ - sartorius branch to sartorius muscle; SM — sartorius muscle; ASIS - anterior superior iliac spine; SCIA - superficial circumflex iliac artery; SEA - superficial epigastric artery; EPA — external pudendal artery.

cutaneous veins and cutaneous nerve was observed in each side of all cadavers.

Body heights and anatomic measurements of the PB and CB based on medial thigh fasciocutaneous flap

The mean body height of the cadavers was 172.70 $(182.70-155.50) \mathrm{cm}$. The diameter of the PB at origin point was $0.14(0.10-0.21) \mathrm{cm}$. The diameter of $C B$ at the piercing point of fascia lata was 0.09 (0.07$-0.12) \mathrm{cm}$. The pedicle length or in other words the total length of $P B+C B$ was $4.74(3.40-6.20) \mathrm{cm}$. The mean length of $C B$ was $3.30(1.80-6.00) \mathrm{cm}$. The other measurements taken to determine the $\mathrm{PB}$ and CB were also given in Table 1.

\section{DISCUSSION}

Medial thigh flaps based on branches of the femoral artery are used in local or extensive skin defect

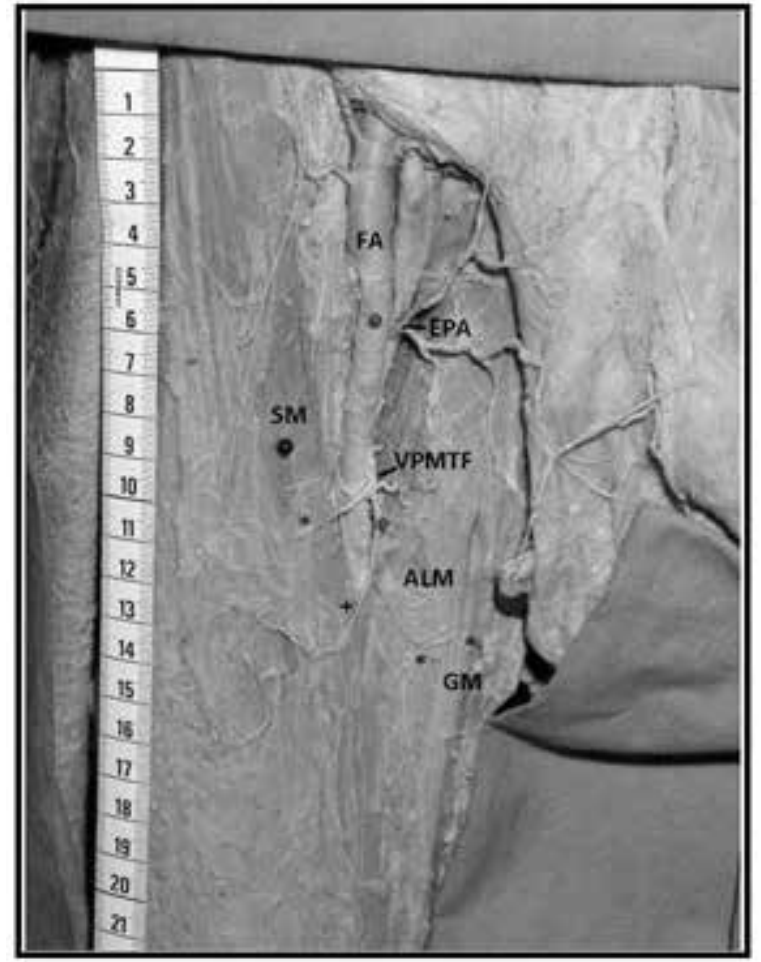

Figure 2. Branches of the femoral artery (FA) were shown emerging along the borders of sartorius muscle to supply the skin; EPA - external pudendal artery; VPMTF — vascular pedicle of the medial thigh fasciocutaneous flap and its muscular $\left({ }^{*}\right)$ (to sartorius, adductor longus and gracilis muscles) and cutaneous branches $\left({ }^{+}\right)$; ALM — adductor longus muscle; GM — gracilis muscle; SM sartorius muscle.

reconstructions. Particularly, flaps were preferred during reconstructive surgery of the thigh and perineal defects, total pelvic exenteration [3], benign or malign scrotal and vaginal tumour excision, radiotherapy related defects $[18,19,21]$ in addition to burn contractures, avulsion, trauma contractures of leg [18], and sex conversion by vaginoplasty in transsexual [3]. The cutaneous and PBs of the femoral artery are valuable for the fasciocutaneous medial flap elevated from thigh region because of its rich and convenient supplying. A vascularised flap is not always necessary during reconstructive surgery, such a choice often leads to a successful outcome and may be rapidly elevated from a single extremity in a single stage that leaves virtually no donor-site deficit and results in better healing with minimal surgical procedure [19]. Hence, we investigated the anatomical features of the perforator and the CBs of the femoral artery which supply to medial thigh flap in details. As recently described by Benninger [2], there is an inconsistency 
Table 1. Anatomic measurements of the perforator and cutaneous branches based on medial thigh fasciocutaneous flap

\begin{tabular}{lccc}
\hline Description & Mean [cm] & Minimum [cm] & Maximum [cm] \\
\hline Diameter of PB (at origin) & 0.14 & 0.10 & 0.21 \\
Length of pedicle & 4.74 & 3.40 & 6.20 \\
Diameter of CB (at origin) & 0.09 & 0.07 & 0.12 \\
Length of CB & 3.30 & 1.80 & 6.00 \\
Distance between origin of PB and ASIS & 18.11 & 12.50 & 24.00 \\
Distance between origin point of PB and IL & 12.16 & 8.00 & 16.50 \\
Distance between origin point of PB and PT & 11.74 & 8.80 & 15.60 \\
Distance between piercing point* of PB and ASIS & 19.55 & 14.90 & 25.00 \\
Distance between piercing point* of PB and IEL & 21.32 & 10.50 & 33.70 \\
Distance between piercing point ${ }^{*}$ of PB and PT & 12.97 & 8.60 & 16.70 \\
\hline
\end{tabular}

*Piercing point is the point where the $\mathrm{CB}$ of the pedicle pierces the fascia lata; $\mathrm{PB}$ - perforating branch of the medial thigh fasciocutaneous flap; $\mathrm{CB}$ — cutaneous branch of the medial thigh fasciocutaneous flap; IL — inguinal ligament; ASIS — anterior superior iliac spine; IEL — inter-epicondylar line; PT — pubic tubercle

or a disagreement about the terminology of the femoral artery and its branches that exists between the anatomical and clinical arenas. The external iliac artery passes beyond the inguinal ligament, and it becomes the femoral artery. The femoral artery gives off the profunda femoris artery (or deep femoral artery) and extends as the femoral artery throughout its course in the proximal two-thirds of the thigh [2].

The femoral artery, in general, and as described by Benninger [2], is the extension of the external iliac artery and gives off the profunda femoris [6]. On the other hand, the clinicians refer to the femoral artery as the superficial femoral artery and its branch as the deep femoral artery. Another confusion on the terminology used by the clinicians and the surgeons is described by Muresian [15], where he uses the termination of the common femoral artery instead of the femoral artery and the branches of the artery as the superficial and the deep femoral arteries. All this knowledge regarding the terminology and nomenclature of the femoral artery and its branches are not recorded in Terminologia Anatomica [6]. A novel agreement on the terminology and nomenclature is needed here for both the clinical arenas and anatomy. In our study, we preferred to use the current and classical terminology according to the Terminologia Anatomica records, where we used the deep femoral as a terminal branch of the femoral artery on the anterior thigh region [6].

Also, we aimed to evaluate the location and origin of the perforator and $C B$ based on the medial thigh pedicle in addition to comprehensively illustrate its vascular pattern for the contribution towards a safe surgical intervention. As it is known among the surgeons, it is important to find out the anatomical landmarks of the thigh region in order to define the location of the pedicle, the suitable flap size and also a feasible vascular pattern for the medial thigh fasciocutaneous flap [19]. In the present study, the length of the pedicle was defined and the vascular evaluation was investigated in order to help the surgeons to optimise the different reconstructive surgical procedures in future.

The possibility of raising the fasciocutaneous flaps from the medial thigh based on perforators which were branches of the femoral artery was firstly described by Beak [1]. The direct cutaneous vessels arising from the femoral artery were the basis of several conventional and less well-known flaps [4]. In the literature, there were limited studies about the significance of the PB and CB of the femoral artery [14]. So, in the present study some topographic anatomical features of the unnamed PB from the femoral artery to the medial thigh pedicle were described in details for the first time.

The sartorius muscle is mainly supplied by the multiple branches of the femoral artery. According to the literature, these branches of the femoral artery may provide an ideal vasculature pattern for the medial thigh flap. In approximately $78 \%$ of the human there was the perforators which were based on the medial thigh flap, which generally is the largest of all perforators, with further passing round the superior or medial border of the sartorius in the apex of the femoral triangle. The larger perforators were placed between $21 \mathrm{~cm}$ and $30 \mathrm{~cm}$ above the plane of the 
knee joint and then they were passing around the medial border of the sartorius muscle [4]. Koshima et al. [11] also defined that the medial thigh flaps were based on the perforators originating from the femoral artery. In the present study, both the CB and PB which were defined to appear in the apex of the femoral triangle and originating from the femoral artery were shown in all of the cases. They were emerged posterior to the sartorius muscle as previously presented $[4,11]$. Also, the $C B$ of the femoral artery was measured as $0.5-1.2 \mathrm{~mm}$ in diameter and its origin was measured up to $1.5 \mathrm{~mm}$ [4]. Feng et al. [7] claimed that the medial thigh perforator flap was supplied by the septocutaneous vessels or the musculocutaneous perforators arising from the superficial femoral artery. The pedicle was $0.7-1.5 \mathrm{~mm}$ in diameter, while, we found in our study that the mean diameter of the pedicle was $0.90(0.70-1.20) \mathrm{mm}$. Our results were in concordance and within the same range of the previously found results $[4,7]$.

Peek et al. [17] found that after distributing off the branches to the adductor longus muscle, the pedicle of the medial thigh flap pierced the gracilis muscle at a location 9-12 cm distal to the pubic tubercle. Hallock [9] described the gracilis musculocutaneous perforators using audible Doppler sonogram at a point approximately $10 \mathrm{~cm}$ below the pubic tubercle and just posterior to the palpable tendon of the adductor longus muscle. In agreement with previous findings $[9,17]$, the location of the medial thigh flap vascular pedicle was at a range $8.80-15.60 \mathrm{~cm}$ distal to the pubic tubercle, giving a mean value of $11.74 \mathrm{~cm}$. Our approach was different from previous studies that we provided a more detailed insight view on different additional surgical reference points. We described the location of the VPMTF related to the reference points such as the anterior superior iliac spine, inguinal ligament and pubic tubercle. Additionally, we described the location of the $C B$ at its origin point, where a branch of the pedicle pierces the fascia lata to supply the covering skin, related to the reference points such as the anterior superior iliac spine, inter-epicondylar line and pubic tubercle. Thus, we aimed to obtain a detailed anatomical knowledge on the perforating and $C B$ of the femoral artery which supplied the upper medial thigh region. Therefore, the results of the present study may play major role during intraoperative periods and postoperative survive while elevating and moving flaps.
Regularly, it is significant to define size, length and harvesting area of the pedicle while elevating the flap and determining the different appropriate incision types [4]. Thus, the borders of the potential skin territory of the medial thigh are reliable and the scar from the closure of the donor site within the medial thigh may be easily concealed by clothing $[19,21]$. The surgeons usually make out surgical landmarks for moving the flap borders [4]. In the present study, we measured the distances between the origin point of the unnamed perforating branch to the medial thigh pedicle and the important surgical reference points in order to provide the optimal standard criteria during surgery. We also provided the emerging point of the perforating branch in the proximity of the femoral triangle apex. Within this context we found that the pedicle of the medial thigh flap was placed on up to $25 \mathrm{~cm}$ from the anterior superior iliac spine in average.

When performing surgical interventions, careful dissections should be performed in this area. Our findings may also provide additional anatomical information, guidance during the process of surgical procedure designing in addition to the optimisation of the surgical intervention procedure.

\section{CONCLUSIONS}

Finally, we can say that during surgical interventions careful dissections should be performed in the pedicle region. Our findings may also provide additional anatomical in $\neg$ formation, guidance during the process of surgical procedure designing in addition to the optimisation of the surgical intervention procedure.

\section{ACKNOWLEDGEMENTS}

The authors thank Ramazan Sayın and Musa Tok, Dokuz Eylul University School of Medicine for their great supportive contribution in form of skilful technical. Also, we would like to acknowledge the efforts of Assistant Prof. Dr Harun. M. Said from the Molecular Medicine Department, Graduate School of Health Sciences at Dokuz Eylül University for his help during the revision process of this manuscript.

\section{REFERENCES}

1. Baek SM (1983) Two new cutaneous free flap, the medial, and lateral thigh flaps. Plast Reconstr Surg, 71: 354-365.

2. Benninger B (2014) Novel femoral artery terminology: integrating anatomy and clinical procedures leading to standardized intuitive nomenclature. Clin Anat, 27: 1085-1088. 
3. Cairns TS, de Villiers W (1980) Vaginoplasty. S Afr Med J, 57: 50-55.

4. Cormack GC, Lamberty BGH (1994) The arterial anatomy of skin flaps. 2nd Ed. "The blood supply to the skin by regions" and "The vascular territories and the clinical application to the planning of flaps". Churchill-Livingstone, Edinburgh, pp. 131-275, 278-439.

5. Ellabban MG, Townsend PL (2003) Single-stage muscle flap reconstruction of major scrotal defects: report of two cases. Br J Plast Surg, 56:489-493.

6. Federative Committee on Anatomical Terminology (FCAT) (1998) Terminologia Anatomica, Thieme Stuttgart, New York, pp. 90.

7. Feng $\mathrm{CH}$, Yang JY, Chuang SS, Huang CY, Hsiao YC, Lai CY (2010) Free medial thigh perforator flap for reconstruction of the dynamic and static complex burn scar contracture. Burns, 36: 565-571.

8. Guan WX (1988) Application of a free medial thigh flap to the treatment of late postburn cases. Zhonghua Zheng Xing Shao Shang Wai Ke Za Zhi, 4: 258-317.

9. Hallock GG (2003) The gracilis (medial circumflex femoral) perforator flap: a medial groin free flap? Ann Plast Surg, 51: 623-626.

10. Jones JW1, Barone RM, Adamson RM (2005) Combined thigh flap for closure of massive trunk defect. J Reconstr Microsurg, 21: 441-445.

11. Koshima I, Hosoda M, Inagawa $K$, Moriguchi $T$, Orita $Y$ (1996) Free medial thigh perforator-based flaps: new definition of the pedicle vessels and versatile application. Ann Plast Surg, 37: 507-515.
12. Lockwood TE (1988) Fascial anchoring technique in medial thigh lifts. Plast Reconstr Surg, 82: 299-304.

13. Malikov S, Valenti P, Masquelet AC (1999) Neurocutaneous flap of the anteromedial aspect of the thigh with a distal pedicle. Anatomical study and clinical application. Ann Chir Plast Esthet, 44: 531-540.

14. McCarthy JG (1990) Plastic Surgery. Vol. 3. WB Saunders Company, USA, Philadelphia, pp. 4044.

15. Muresian $\mathrm{H}$ (2015) Novel femoral artery terminology: from Inconsistent and confusing to stereostructural clarity? Clin Anat, 28: 424-425. doi: 10.1002/ca.22477.

16. Muruci A, Muniz CR (1987) Nipple reconstruction with muscle flap. Aesthetic Plast Surg, 11: 41-43.

17. Peek $A$, Müller $M$, Ackermann $G$, Exner $K$, Baumeister $S$ (2009) The free gracilis perforator flap: anatomical study and clinical refinements of a new perforator flap. Plast Reconstr Surg, 123: 578-588.

18. Spirito D (1998) Medial thigh lift and DE.C.LI.VE. Aesthetic Plast Surg, 22: 298-300.

19. Strauch B, Vasconez LO, Hall-Findlay EJ (1998) Grabbs's Encylopedia of flaps. 2nd Ed. "The anteromedial thigh flap", "Medial thigh skin flaps for repair of vaginal stenosis", and "Medial thigh skin flaps for reconstruction of scrotum". Lippincott-Raven, Philadelphia, pp. 433-438, 1458, 1575, 1998.

20. Turley CB, Cutting P, Clarge J (1991) Medial fasciocutaneous flap of thigh for release of post-burn groin contractures. Br J Plast Surg, 44: 36-40.

21. Wang TN, Whetzel T, Mathes SJ, Vasconez LO (1987) A fasciocutaneous flap for vaginal and perineal reconstruction. Plast Reconstr Surg, 80: 95-103. 